\title{
Advances in Mechanics and Outlook for Future Mankind Progress
}

\author{
Joseph J. Smulsky \\ Institute of Earth Cryosphere SB RAS, 625000, Tumen, P.O.Box 1230, Russia \\ Email: jsmulsky@mail.ru
}

\begin{abstract}
Three problems for the interaction between N bodies to which exact solutions were obtained are discussed. In the first problem, the bodies are located axisymmetrically in a plane. Possible trajectories of the bodies here are ellipses, parabolas, hyperbolas, or straight lines. Results of solving this problem are further used for solving problems about Earth's rotation and Mercury's perihelion. In the second problem, the body system consists of several layers, and it rotates as an entity. In the third problem, the bodies are uniformly distributed over a sphere, and they move experiencing no mutual collisions. The latter problem allows the formation of several planets, for instance, one hundred planets resembling the Earth and moving under identical conditions with respect to the Sun. The latter possibility opens a way toward unrestricted mankind progress.
\end{abstract}

Index Terms $-N$-bodies problem, exact solutions, Earth axis, Mercury's perihelion, mankind progress.

\section{INTRODUCTION}

There exist problems whose solution predefines further route of the whole mankind. One of such problems is the problem on the interaction of two bodies. As a result of the solution of this problem for gravitational interaction, people have understood why and how bodies move in the cosmic space. Under the action of the Sun, planets move in roughly circular orbits while comets travels in elongated elliptical orbits or, sometimes, along parabolic trajectories. For a space vehicle to be launched to Mars, it is necessary that that vehicle moved relative to the Earth along a hyperbolic trajectory.

The latter knowledge and the related human activity would have never been possible if the problem of two bodies were not solved. Many people are not aware of this fact, and they do not comprehend it. To date, one may come across people, even scientists, who believe that the planets in the Solar system orbit round the Sun due to ether swirling, the Sun acting as a sink or source for ether [1]. In their speculations, such peoples follow the Descartes hypotheses. Today, we are able to comprehend what route would have been followed by mankind unless the two-body problem was not be solved by Isaac Newton.

In the present paper, I invite the reader to consider the solutions to three problems concerning the interaction between bodies. Will it be their impact on the further development of mankind in importance is the same as the two-body problem?

While studying materials on the Easter Island, noted geologist F.P. Krendelev had arrived at a conclusion that the life of the islanders, who were perfectly isolated from the outer world, was guided by 15-20 knowers, who were called by F.P. Krendelev scientists [2]. The latter is also the case for any society at any historical period: science was the guiding star for that society. Mechanics, which is a science that studies the interactions and motions, forms the basis for mankind's productive activity. Let us consider the matter of how results of this science may promote and guide mankind in its evolution.

Earlier, my reflections on mankind's evolution were outlined in several publications [3] - [7]. Here, I discuss one possible evolutionary path that permits solving the problem of $\mathrm{N}$-bodies uniformly distributed round a sphere.

\section{AXISYMMETRIC INTERACTIONS OF N-BODIES ON A PLANE}

The problem of gravitational interaction of two bodies was exhaustively and exactly solved by Isaac Newton more than three hundred years ago. The solution of this problem can also be applied to the Coulomb interaction of two charges. The results form the basis for the main principles of the physics of micro- and macroworld. No other exact and complete solutions to the problems of the interaction between bodies have so far been obtained. In 1996, a second exact and complete solution to an interaction problem was found for a special body configuration that involved $N$ bodies (Fig. 1a) [8] - [10]. Initially, $N-1$ bodies of identical mass $m_{l}$, which have identical transversal $v_{\tau}$ and radial $v_{r}$ velocities, are uniformly arranged in a circumference of radius $R$. At the center, one more body having some mass $m_{0}$ can be located. This problem admits an easy solution. First, the force acting on a body from the side of all the other bodies is to be determined. Then, division of this force by the mass $m_{l}$ yields the acceleration of the body. In fact, the accelerations of all bodies yield differential motion equations for the whole body system. As a result of solution of the equations, one can find the velocities, the trajectories, and the laws of motion of all the bodies. 

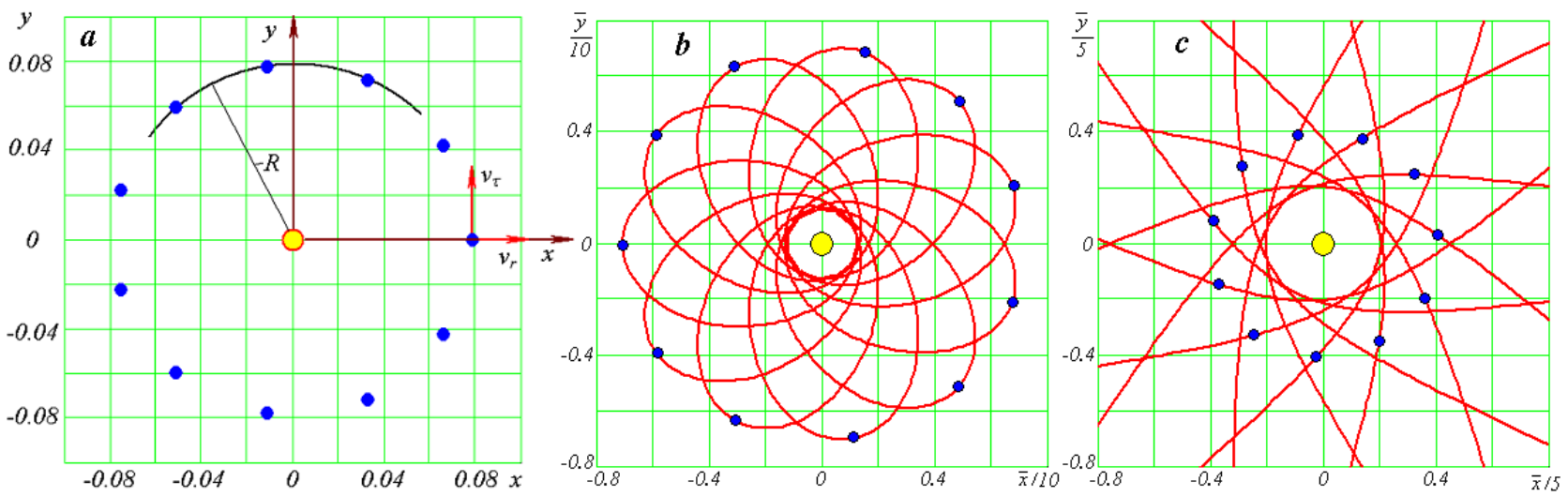

Fig.1. Gravitational interaction of $N$ bodies axisymmetrically arranged in a plane: a - schematic illustrating the solution of the problem for eleven peripheral bodies: $b$ - elliptical motion; $c$-hyperbolic motion; $x$ and $y$ are coordinates in the plane in which the bodies move; $\bar{y}=y / R_{P}$ and

$$
\bar{x}=x / R_{P} \text {, where } R_{P} \text { is the pericenter radius. }
$$

Depending on the initial values of velocities $v_{\tau}$ and $v_{r}$ (Fig. 1a), the bodies move along ellipses contained within a circumference of radius $R$, along a circumference of radius $R$, or along ellipses coming out to distances $r>R$ (see Fig. $1 b$ ). With further increase of velocity $v_{\tau}$, the trajectories become parabolic and, then, hyperbolic (see Fig. 1c). At transversal velocity $v_{\tau}=0$, the bodies move along radii toward the center. All found criterions and laws of motion are valid for all relevant cases [8] - [10].

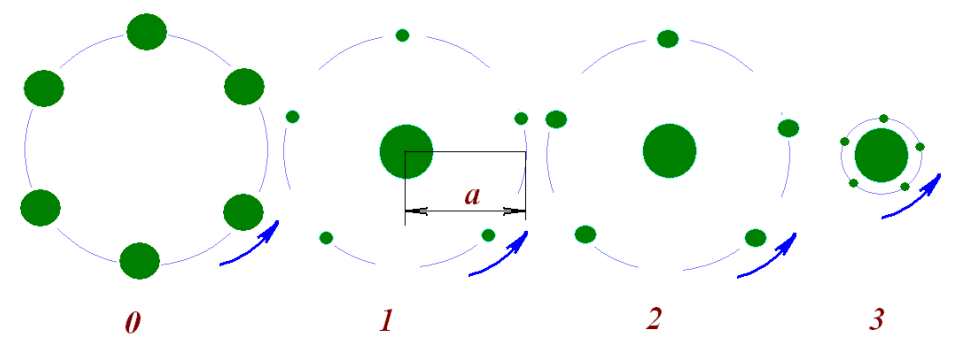

Fig.2. Four compound models for Earth's rotation: 0 - without a central body, $1-3$ - with a central body; $a$ - orbital radius of peripheral bodies. The mass of the model systems is equal to the Earth mass; the evolution of the orbit of a peripheral body models the evolution of the Earth's equatorial plane.

The obtained solution to this problem were used to develop a compound model for Earth's rotation [11], which has revealed specific features of the Earth's rotational motion. Later, this approach has proved helpful in solving the forward problem about the Earth's rotation over a period of hundred thousand years [12] - [14]. As a result, new data concerning the evolution of Earth axis were obtained: the axis was found to experience large oscillations whose amplitude reached $\pm 7-8^{\circ}$. This result has completely explained the periodic alternation of glacial epochs and warm periods [14] - [16].

The compound model of the Sun (Fig. 3) can be used to examine the impact of the Sun's oblateness on the motion of planets [17]. It was found that the latter action brings about an additional rotation of orbit perihelia; this effect is more pronounced the closer the planet resides to the Sun. The most substantial one is Mercury's perihelion rotation. The rate of this rotation, together with the rate of the rotation due to all other planets, well complies with the observed value. Therefore, the explanation to this phenomenon based on the hypothesis of gravity propagation at the speed of light proves to be inadequate.
It is a well-known fact that the latter hypothesis was laid to the basis of the General Theory of Relativity.

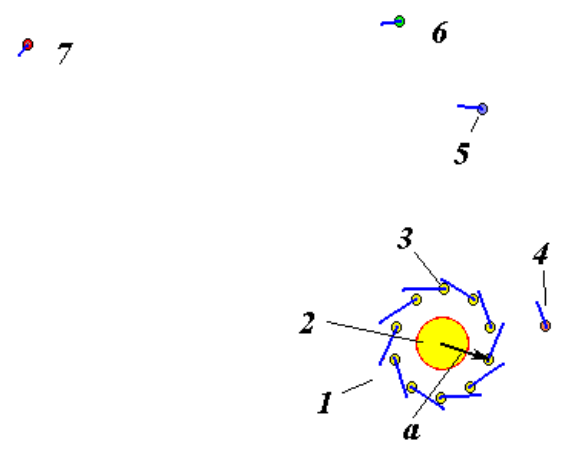

Fig.3. Action of a compound model of the Sun (1) on the Solar-system bodies: 2 - central body; 3 - peripheral body; 4 - Mercury; 5 - Venus; 6 - Earth and Moon; 7 - Mars.

Above, we were speaking of macro-world. The same problem was also solved for the interaction of charged particles axisymmetrically arranged in a plane (Fig. 4): at the center, we have a positively charged nucleus with 
electrons moving at the periphery [18]; here, the particles interact with each other via the Coulomb force. Quite astonishing results were obtained. With increasing the total number of the electrons (and with the nucleus charge varying in proportion to this number), the forces ensuring the attraction of the electrons to the nucleus were found to grow in magnitude as the total number of electrons increased to $\mathrm{Ne}=174$ and, afterwards, those forces weakened as the number of electrons increased to $\mathrm{Ne}=$ 473; subsequently, newly added electrons experienced no attraction to the nucleus [18] - [19]. This result possibly explains why there exist only a finite number of chemical elements in the nature.

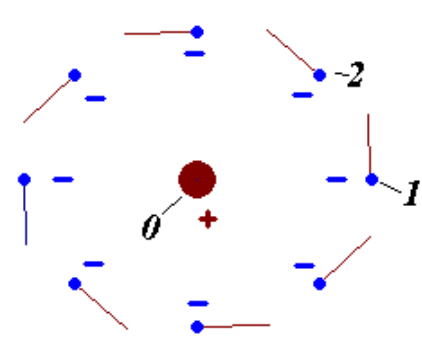

Fig.4. Coulomb interaction of nine particles forming an axisymmetric configuration with parameters taken equal to those for the oxygen atom: $O$ - central particle; 1 and 2 - the first and second peripheral particles.

For computing the parameters of axisymmetric structures, MathCad programs were developed. Those free-access programs are available in the file InCnPrpr.mcd http://www.ikz.ru/ smulski/GalactcW/InCndFls/Preprtn/ (for gravitational interaction) and in the file InCnPrClb.med at http://www.ikz.ru/ smulski/GalactcW/ModCoulm/InCnd $\mathrm{FsQ} /$ (for Coulomb interaction).

\section{MUlti-Layer Rotating StruCtURES INVOLVING N} BODIES

$$
F_{n, j}=\frac{G \cdot m_{j}}{r_{j}^{2}}\left[m_{0}+\sum_{i \neq j}^{N_{2}}\left[m_{i} \cdot \sum_{l=1}^{N_{3}} \frac{1-r_{i, j} \cdot \cos \Delta \varphi_{j, 1, i, l}}{\left(1+r_{i, j}^{2}-2 r_{i, j} \cdot \cos \Delta \varphi_{j, 1, i, l}\right)^{3 / 2}}\right]+m_{j} \cdot f_{n 3}\right] .
$$

In expression (1),

$$
f_{n 3}=0.25 \sum_{l=2}^{N_{3}} \frac{1}{\sin \left[\pi(l-1) / N_{3}\right]}
$$

$G$ is the gravitation constant;

$r_{i, j}=r_{i} / r_{j}$ is the ratio between the radii of the $i$-th and $j$-th rings;

$\Delta \varphi_{j, 1, i, l}=\varphi_{j, 1}-\varphi_{i, l}$ is the angular difference between the radii of the bodies $j, 1$ and $i, l$;

$F_{n, j}$ is the force, directed along the normal $n$ in the trajectory coordinate frame $n, \tau$.

For constructing the rotating systems, a program RtCrcSt2.for was developed [20]; this program can be accessed at www.ikz.ru/ smulski/Data/RtCrcStr/. Structures with different total numbers of layers $N_{2}$ and with different total numbers of bodies in the layers $N_{3}$ were obtained. The total number of the bodies, $N$, varied from three to million bodies. Three structures with fifteen layers and 30 bodies in each of the layers at various arrangements of particles in layers are shown in Fig. 6. The configurations of the structures differ in the angle $\varphi_{j, 1}$ 
of the first body. The numbers in the table show the bodies in the layers. relative radii of the layers and the relative masses of the
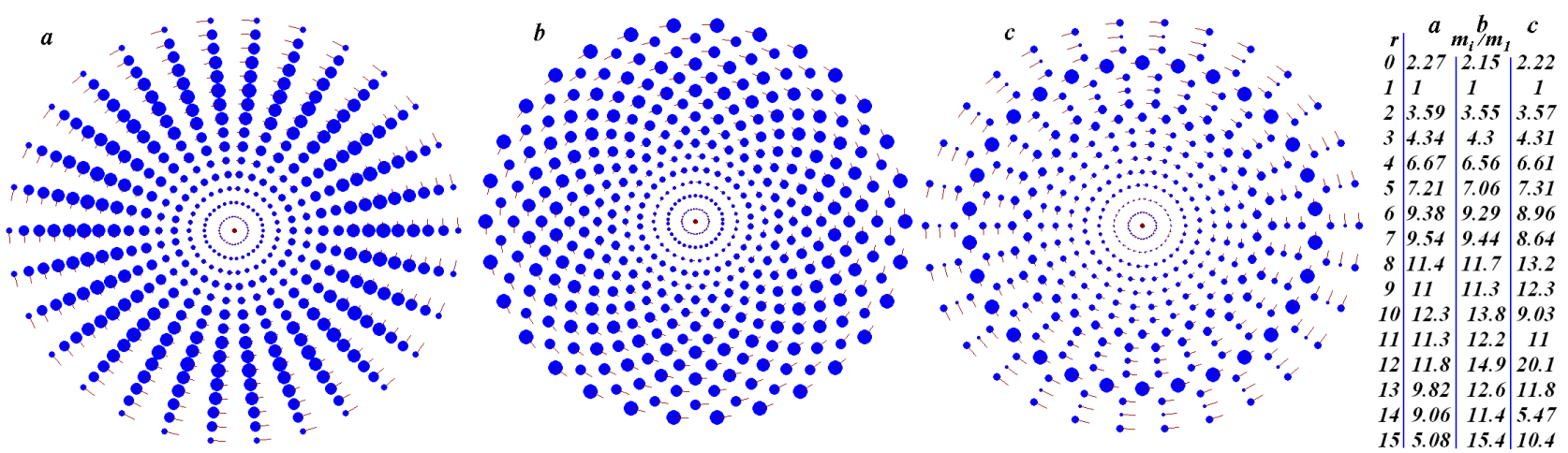

Fig.6. Images of axisymmetric multi-layer rotating structures as displayed on the computer screen. The structures were obtained by integration of differential motion equations performed using the Galactica program: $N_{2}=15 ; N_{3}=30$; period $P_{r d}=1$ year; the mass of the central body is equal to the Sun mass. Given in the table are the relative radii of the rings and the masses of one body in the rings.

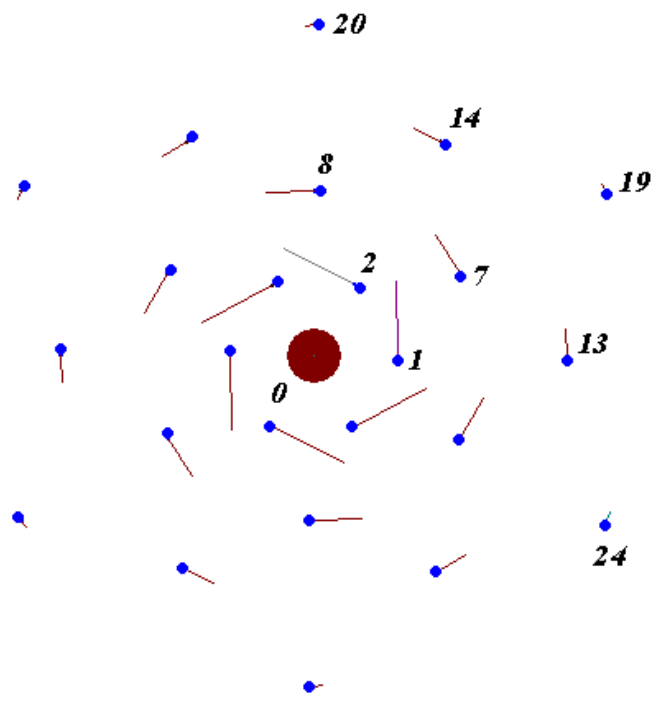

Fig.7. A four-layer structure with the Coulomb interaction between particles: 0 - positively charged nucleus; 1 - 2 - particles forming the first layer; 7 - 8 - particles forming the second layer; 13 - 14 - particles forming the third layer; 19, 20 and 24 - particles forming the fourth layer.

The same problem was formulated and solved for the Coulomb interaction [19], the solution in that case, however, being non-existent. In all multi-layer structures, the repulsive forces of the electrons proved to be stronger than the attraction of the electrons to the nucleus. This result seems to be of fundamental importance: for atoms, no planetary model rotating as a whole is possible. The obtained results have helped us in developing atomic models with differential rotation of electron layers (Fig. 7).

The developed programs allow the researcher to construct any structures, obtain solutions for those structures, and examine the dynamics and evolution of the structures using the Galactica system. Methods to investigate into the dynamics and evolution of the structures of interest were developed in [18] - [20].

\section{STRUCTURES OF N BODIES REGULARLY DISTRIBUTED IN SPACE}

The above structures exist on a plane. A further question is: is it possible to obtain a spatial structure exercising a regular motion?

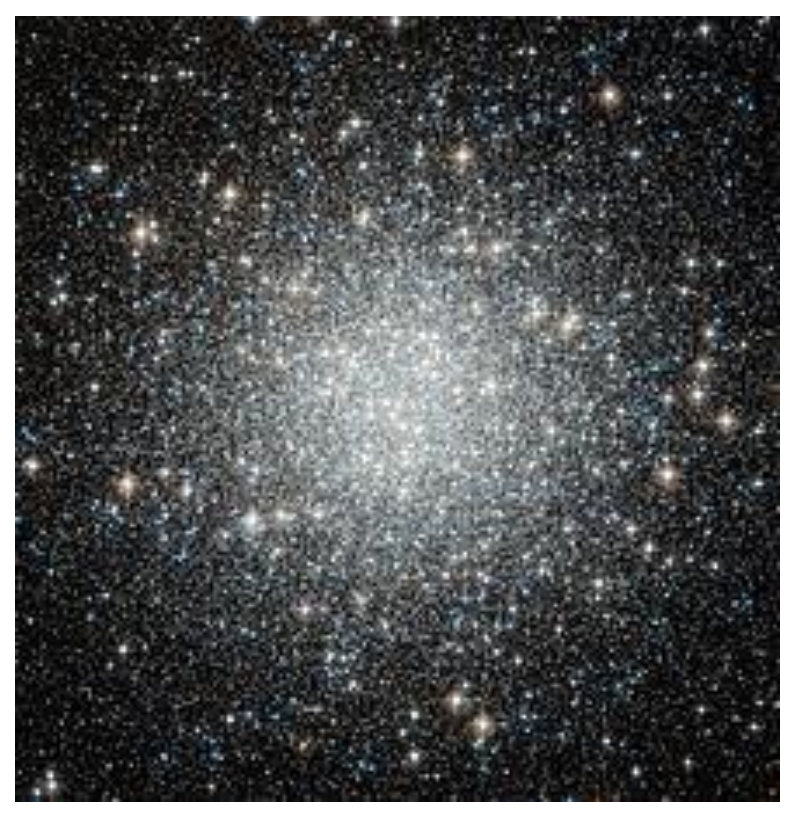

Fig.8. The M 53 (or NGC 5024) globular star cluster in the Coma Berenices constellation (https://ru.wikipedia.org/wiki/M_53).

Spatial structures need to be studied for gaining insight into the nature of globular star clusters (see Fig. 8). In such clusters, all stars attract to one another; yet, they do not merge together to form a superstar nor they collapse with the passage of time. Why does this so occur?

By deploying individual body orbits in a planar axisymmetric structure out of a single plane (see Fig. 1a), one can obtain a spatial structure. However, there is an infinite number of possible ways to transform a planar structure in a spatial structure. We tested many of such ways to identify a most appropriate strategy. By this 
method, one can construct a structure whose bodies will exercise an eternal regular motion. Such a structure involving 99 peripheral bodies is shown in Fig. 9. Those bodies are arranged in a line making three turns on a sphere (Fig. 9b). Each of the peripheral bodies travels in its own circular orbit. Here, the distances between the bodies remain unchanged, so that the bodies never collide with each other.
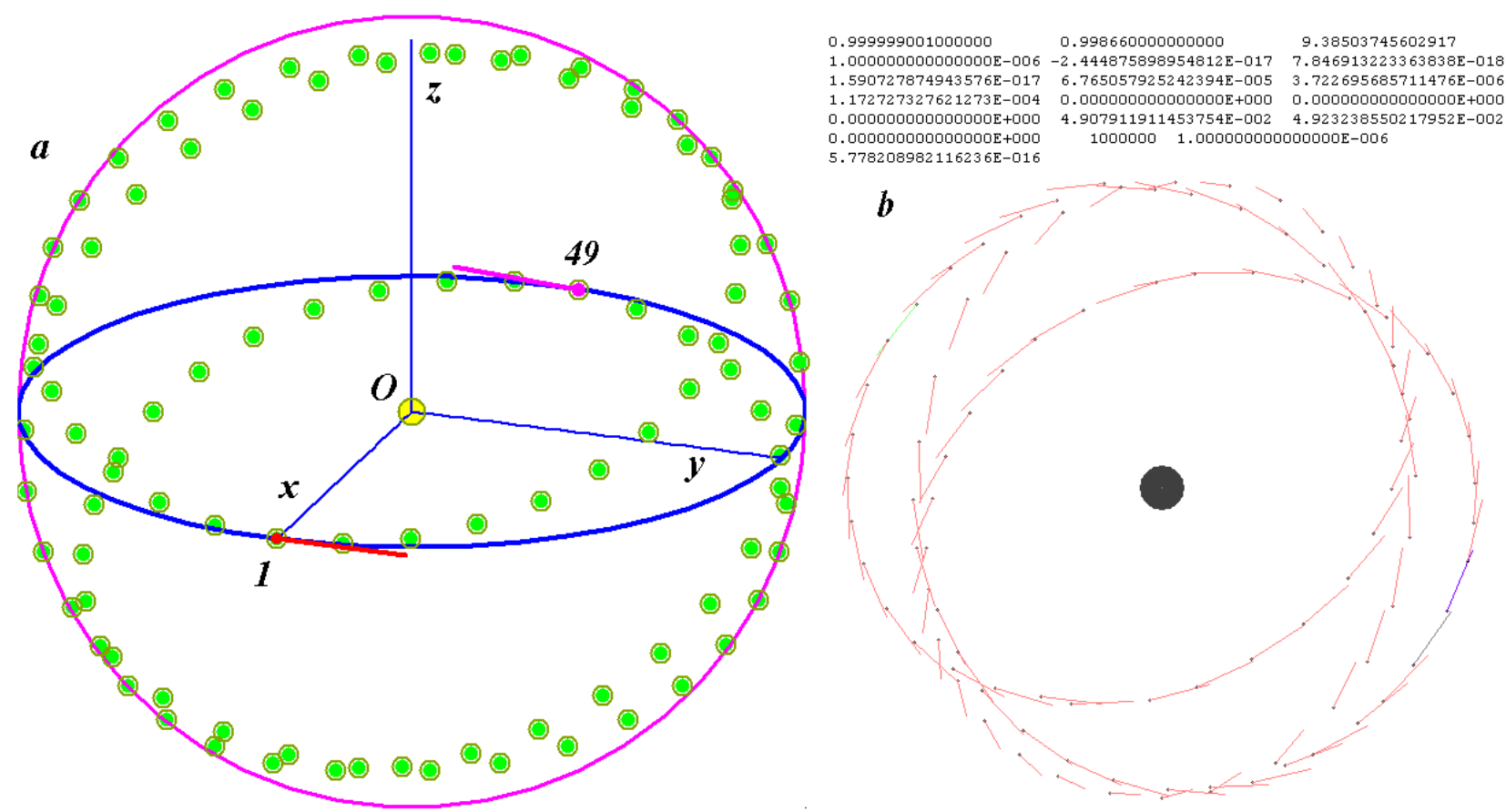

Fig.9. A regular spatial structure with $N=100, R=1$ AU (astronomical unit is equal 1.496 million $\mathrm{km}$ ), and period $P_{r d}=1$ year and with the centralbody mass equal to the Sun mass: $a$-in 3D coordinate system, the lines at bodies 1 and 49 indicate the velocity vectors; $b$-image of the structure as displayed on the computer screen after 100 revolutions of a peripheral body around central body in the integration of the differential motion equations with the Galactica system; $a$-projection onto the frontal plane, $b$ - projection onto the horizontal plane.

For numerical integration of the differential motion equations of 100 bodies in the above structure by the Galactica system, we specified a central-body mass equal to the Sun mass and took the mass of the peripheral body equal to the Earth mass. The distance of the bodies to the Sun was the same like that of the Earth, and their orbital period $P_{r d}$ was taken equal to the Earth year. The system proved to be stable, with no indications for its changes having emerged after one hundred revolutions of the peripheral bodies. Indicative of the latter are the numerical data shown in Fig. $9 b$ [21].

An algorithm and a program for calculating such structures were developed [22]. Any configurations of the structures with any body masses and system sizes could be obtained. Since the motions of the bodies in the structures are regular, they can be represented with analytical expressions. Those expressions define the position and velocity of each body at any time. In fact, they present exact solutions to the $N$-body problems of special type.

Thus, a third exact solution to the $N$-body problem was obtained; unlike in the first two problems, here we have a spatial solution. It should be noted that the developed algorithm permits construction of structures in which the bodies will move along ellipses, parabolas, or hyperbolas.

\section{FORMATION OF THE FUTURE HABITAT FOR MANKIND}

The existence of a solution that shows that, on a sphere with a radius equal to the Earth's orbit radius, there can be several planets identical to the Earth offers new perspectives for further mankind evolution. New planets can be created from the matter available in the Solar system. That matter can be the substance that presently forms asteroids, planet satellites, and planets themselves. New planets can be created successively. First, a second planet (following the Earth) is to be created. This planet is to be populated with primitive life forms and, after those life forms become well-rooted, more advanced plant and animal types are to be introduced. This process is to be continued unless conditions suitable for human life are established. From this time on, a long-term stage of human dissemination and dissemination of the whole living word over the planet is to be initiated.

Life originated on the Earth as a result of multiple random interactions. That is why it took several billiard years for life to emerge and evolve to its present form. As a result of conscious human activities, the origination of life and its evolution on a new planet will proceed much faster, taking, instead of several billiard years, just several hundred years. 
After development of the second planet, actions aimed at the creation of a third planet and, then, all subsequent planets can be initiated. With introduction of each new planet, the orbits of the previously formed planets are to be corrected in such a way that to allow all planets available in the system by that time to execute a regular motion, that is, to conform to the exact solution of the spatial problem for the given number of involved bodies.

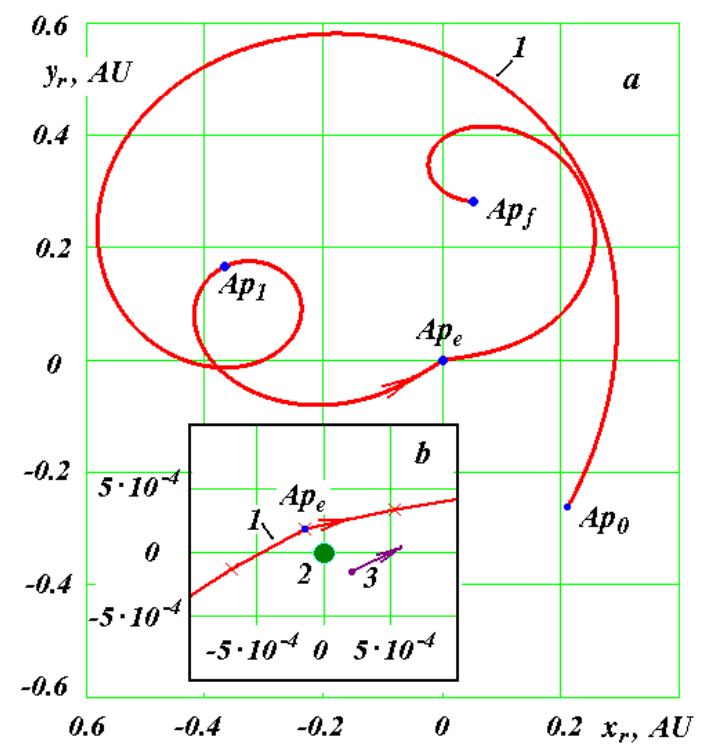

Fig.10. Asteroid Apophis' trajectory (1) relative to the Earth (2): $a$ - on the traditional scale, $b$ - on an enlarged scale by the moment of Apophis' approach to the Earth (2); 3 - Apophis' position at the moment of its approach to the Earth after trajectory correction; $A p_{0}$ and $A p_{f}$ are the initial and end points of Apophis' path segment; $A p_{e}$ is the point of Apophis' closest approach to the Earth; $A p_{I}$ is the trajectory

correction point; the coordinates are given in astronomical units.

For creation of a new earth, mankind has to gain sufficient experience in managing with cosmic objects. In 2008, we have suggested turning asteroid Apophis into an earth satellite [23] - [24]. On April 13, 2029, the asteroid will approach the Earth to a distance of six Earth radii (see Fig. 10); no such approaches will subsequently occur during a period of the next thousand years. It would be desirable that the satellite Apophis would rotate round the Earth in the same direction like the Moon does. To this end, half a year before the approach (point $A p_{1}$ ) Apophis needs to be slightly accelerated so that to bring it to the night side of the sky. Deceleration will be required at the moment Apophis will most closely approach the Earth (point $A p_{e}$ ), at point 3 .

Another scenario can be implemented while making a larger 1950 DA asteroid in the Earth's satellite (see Fig. 11) [23] - [24]. For making that asteroid to round the Earth orbit, we have to accelerate the asteroid at its aphelion point $\left(A_{a}\right)$ and, then, decelerate it at the point of its closest approach to the Earth (that is, at the point $A_{b}$ ). Following this, the 1950 DA asteroid will become an Earth satellite moving at a geostationary orbit.

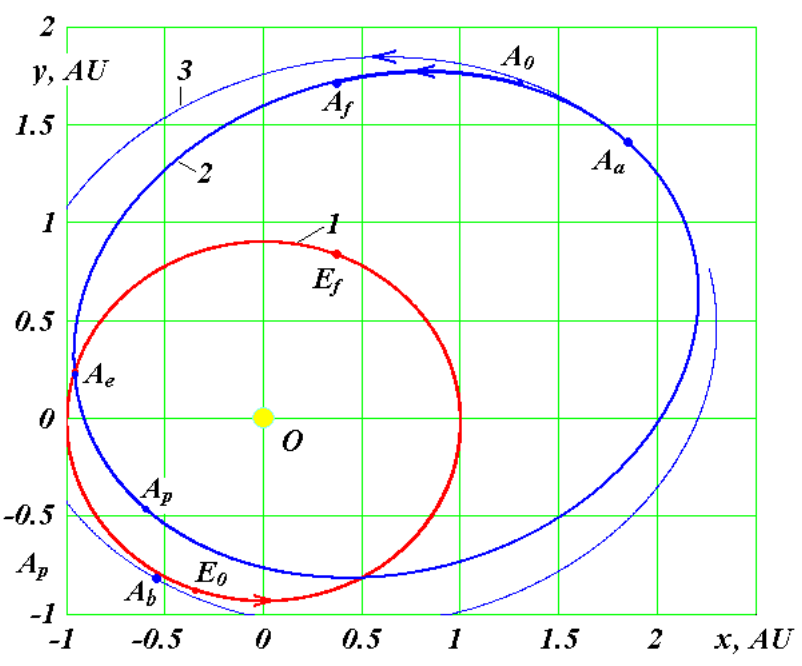

Fig.11. Trajectories of the Earth (1) and 1950 DA asteroid (2) with respect to the Sun (point $O$ ) in baricentric equatorial frame $x O y$ over a period of 2.5 years in the approach epoch of March 6, 2641 (point $A_{e}$ ): $A_{0}$ and $E_{0}$ are the initial points of the path segments of the asteroid and Earth, respectively; $A_{f}$ and $E_{f}$ are the end points of their paths; $A_{a}$ and $A_{p}$ are the aphelion and perihelion points of the asteroid orbit; $3-1950$ DA asteroid orbit after its correction at the point $A_{a}$ is shown conventionally.

Turning asteroids into Earth's satellites are projects easier to accomplish in comparison with the creation of a new earth. However, today such projects still present tasks technologically impossible to implement. The proposal on Apophis for the year of 2029 made in 2008 had a 21-year leeway. While acting purposefully, mankind could cope with even more cardinal tasks. For instance, after the destructive war it took twelve years for the Soviet Union to successfully launch the first sputnik and sixteen years, to launch the manned spaceship. Those projects were implemented by just one country. It can hardly be possible to imagine what achievements can be made in a period of 21 years providing that the whole mankind will unite the peoples' efforts in solving this problem.

Turning asteroids into the Earth's satellites is a goal for the whole mankind rather than for a single country or private industry. The task on accomplishing this goal implies opinion consistency of all states, peoples, social groups, and individuals. Falling out of step in such understanding will entail a catastrophe that would present a danger for all people.

The present-day mankind is still far from having reached such understanding. In the epoch of the Soviet Union, the country was dominated by the feelings of good-neighborliness, mutual readiness to help, fraternity, and sheared great purposes for space exploration and hopes for establishing links and contacts with extraterrestrial civilizations. Nowadays, mankind has plunged into obscurantism, reaching domination over opponents, lie and falsehood, struggle and conflicts between the states and different social groups as well as between individuals. In such a community, mankind fails to succeed not only in achieving adequate goals but even in formulating them. 
Yet, there exist some primal factors that define the mentality and activity of both mankind as an entity and its individual members. As a rule, people remain unaware of the existence of such primal factors, and very seldom can those factors be identified by somebody. However, such factors form motivation for all acts and deeds of humans.

For the current mankind, one such factor is the tightness of our mother Earth and the limited resources available on it. That is why the misanthropic ideology, causing troubles for the states, social groups and individuals, has presently rooted throughout the world to obsess everyone and make him grasp more and more property while leaving nothing to others, to crudely suppress other people, and so on. Those cruel ideas have led our world to many other negative phenomena inherent to our epoch. Cities grow folly while rural areas rapidly lose population. Previous great achievements gained by peoples under the guidance of great leaders have turned condemned and abandoned. Chaos, bigotry and poverty are each and every day introduced into our life in the guise of democracy.

Now, with the obtained exact solution of the problem about regular spatial interaction of $N$ bodies, we can say: «Calm, people, do calm! There will be enough place for all of you under the Sun! We can create more earths. On each earth, we will be able to build a happy and exciting life, not only for people but, also, for plants and animals».

Presently, mankind faces great tasks and problems that can only be accomplished and solved by joint efforts of all peoples. Adequate comprehension and awareness of this fact will lead us to harmonic relations between states and peoples. Time will pass, and new people, when turning back to look at our epoch, will be confused while trying to understand how we could come up to such barbarism and savageness.

\section{DISCUSSION}

The above material was reviewed by three journals for publication. Their opinions are the discussion of the paper by other people. I think they are of interest to the reader.

Eva Scalzo, Editor of Icarus, accept the following decision (June 14, 2016): "Thank you for submitting your manuscript to Icarus. Your manuscript is not appropriate for publication in Icarus and will not be sent out for review. Your paper is more appropriate to an astrophysical or celestial mechanics journal".

Sylvio Ferraz Mello, Editor in Chief, Celestial Mechanics and Dynamical Astronomy (June 24, 2016) sent me such decision: "On this occasion I regret to inform you that Celestial Mechanics is unable to accept your manuscript for publication. We sometimes receive high-quality submissions that cannot be accepted due to limitations in space, scope, or other factors. With this in mind, I would like to offer you the option of transferring this submission to SpringerPlus, where I think your manuscript would be well-suited".

Simon Jones, Editor of electronic journal "SpringerPlus", inform me (September 15, 2016): "Peer review of your manuscript is now complete and, in the light of the reports, and my own assessment as Editor, I regret to inform you that your manuscript cannot be accepted for publication in SpringerPlus".

The review has major comments and specific comments.

\section{Reviewer's major comments:}

The manuscript aims to be a regular research paper. However, it is rather constructed as a review of the Author's ideas spread over literature embedded with some long-term visions about possible applications of these particular ideas. It's impressive how easy the manuscript transforms periodic solutions in the ideal $\mathrm{N}$-body point mass model to technological, philosophical, demographic, political and futuristic matters. In this sense I would consider this as a general essay, and not a research article. I doubt this form of presentation and the conclusions are suitable for Springer Plus and deserve publication as a peer review paper. I would like to note that the final part, considering speculations about building a number of Earth-like planets from other Solar system bodies to provide new habitats for the mankind must be considered rather science-fiction. And a further discussion about this part could be a matter of hand-waiving rather than a solid research.

A major problem is a lack of citations to the well known and settled results of the "official" science which are challenged by the Author's results and research. The bibliography list is a festival of self-citations - the list has about of 25 items, and only three of them are not the Author's papers. In this sense, besides speculations and a general discussion in the last part of the manuscript, the paper does not bring any new result.

Besides these general comments, I found a number of issues that make me worry about the scientific contents of the paper.

\section{Reviewer's particular comments:}

1) The Author claims that "In 1996, a second exact and complete solution to an interaction problem was found for a special body configuration that involved $\mathrm{N}$ bodies (Fig. 1a) [8] - [10]". However, many variations of the $(N+1)$-body configurations composed of a central mass and N-body ring structure have been investigated by Kalvouridis (a list is easily available through ADS database, http://adsabs.harvard.edu/abstract_service.html), back to 1998. Particular periodic solutions to the N-body problem with equal masses are known as n-body choreographies, as originated in 2000 by Chenciner and Montgomery. One such orbit is a circular orbit, with equal masses at the corners of an equilateral triangle (Lagrangian solution, known back to 1772); another is the figure- 8 orbit, first discovered numerically in 1993 by Cristopher Moore and subsequently proved to exist by Chenciner and Montgomery. Choreographies can be constructed by variational methods, as explained by Simo and Chencinier, please see, for instance, https://www.math.uni- 
bielefeld.de/ rehmann/ECM/cdrom/3ecm/pdfs/pant3/sim o.pdf.

2) In neither case the N-body solutions are shown dynamically stable for 3-4 Gyrs (regarding, for instance, the Earth composition model). Moreover, in page "The obtained solution to this problem were used to develop a compound model for Earth's rotation [11], which has revealed specific features of the Earth's rotational motion. Later, this approach has proved helpful in solving the forward problem about the Earth's rotation over a period of hundred thousand years [12] - [14]. As a result, new data concerning the evolution of Earth axis were obtained: the axis was found to experience large oscillations whose amplitude reached $\pm 7-8^{\circ}$. This is not a new result, either, see, for instance a remarkable paper by by Laskar et al. (Laskar, J., Joutel, F., Robutel, P. [1993]. Nature 361, 615-617), who shown that the Earth's Moon stabilizes Earth's obliquity such that it remains within a narrow range, between $22.1^{\circ}$ and $24.5^{\circ}$. Without lunar influence, a frequency map analysis by Laskar et al. (Laskar, J., Joutel, F., Robutel, P. [1993]. Nature 361, 615-617) showed that the obliquity could vary between $0^{\circ}$ and $85^{\circ}$. Further, Lissauer, Barnes and Chambers (Icarus, 2011) have shown that while obliquity varies significantly more than that of the actual Earth over 100,000 year timescales, the obliquity remains within a constrained range, typically $20-25^{\circ}$ in extent, for timescales of hundreds of millions of years. Therefore, the Author should better explain and justify a statement in p. II.: "This result has completely explained the periodic alternation of glacial epochs and warm periods [14] - [16]". Does the Author's model account for the presence of Moon, tidal effects (that lead to measurable changes of the Earth rotation and the semi-major axis of the Moon, and other "subtle" physical factors and circumstances?

3) "The compound model of the Sun (Fig. 3) can be used to examine the impact of the Sun's oblateness on the motion of planets [17]. It was found that the latter action brings about an additional rotation of orbit perihelia; this effect is more pronounced the closer the planet resides to the Sun" (p. . II.). It is well known that non-point and/or non-Newtonian gravitational potentials imply the precession of satellite orbits, hence one would be probably not surprised that a particular mass distribution leads to such an effect (moreover, one of the well known laws of logic says that a false premise may imply true conclusions). "Thus, the explanation to this phenomenon based on the hypothesis of gravity propagation at the speed of light proves to be inadequate. It is a well-known fact that the latter hypothesis was laid to the basis of the General Theory of Relativity." I do not understand what new the Author's model brings here, since the precession of Mercury's orbit is fully explained by the GR, which after 100 years remains unchallenged by any observational test.

4) "In such clusters, all stars attract to one another; yet, they do not merge together to form a superstar nor they collapse with the passage of time. Why does this so occur?" (p. IV.). I would recommend to study - I admit an endless list of papers regarding these matters by, for instance, Douglas Heggie, Sverre Aarseth, Miroslaw Giersz (please see the ADS database) and references in these papers for over the past half-century. Dynamical interactions and evolution of globular clusters is one of the leading subjects of contemporary astronomy, especially armed with high-speed computers.

5 ) "For numerical integration of the differential motion equations of 100 bodies in the above structure by the Galactica system, we specified a central-body mass equal to the Sun mass and took the mass of the peripheral body equal to the Earth mass. The distance of the bodies to the Sun was the same like that of the Earth, and their orbital period was taken equal to the Earth year. The system proved to be stable, with no indications for its changes having emerged after one hundred revolutions of the peripheral bodies. Indicative of the latter are the numerical data shown in Fig. $9 b$ [21]." (p. IV.). There is no real proof that such a system may remain stable for evolutionary time-scale ( $1 \mathrm{Gyr})$, hence for as long as $10^{\wedge} 7,10^{\wedge} 8$, perhaps $10^{\wedge} 9$ revolutions.

6) Regarding the last claim «Now, with the obtained exact solution of the problem about regular spatial interaction of $\mathrm{N}$ bodies, we can say «Calm, people, do calm! There will be enough places for all of you under the Sun! We can create more earths. On each earth, we will be able to build a happy and exciting life, not only for people but, also, for plants and animals». How we could build a number of Earths like planets is a mystery (and science-fiction) as the Author admits. Probably, if the technology will be so advanced, we could transport large moons of Jupiter or large Kuiper-belt objects to the habitable zone, to avoid costs of "assembling" new planets (please note the whole asteroid belt mass is just a tiny fraction of the Earth mass). They wait for us, see a new paper by Batygin and Brown (ApJ, 2016), about newly discovered Planet Nine, and similar large superEarth planets. We could also "just" move Venus and Mars to proper orbits. But, more seriously, the paper does not answer for the most critical part of such a vision, how such a system of planets could be made stable for say 1 Myr. On the other hand, however, if we are able to transport Kuiper-belt objects, or assemble new Earths, or move Venus, it will be not any problem of maintaining these new Earths in proper places.

To summarize, given so speculative character of this paper, and not sufficient scientific justification, also regarding missing references, I do not recommend this manuscript for publication in Springer Plus.

\section{Autor's Response to Reviewer's report}

First, I would prefer to give my answers to the reviewer's particular comments and, then, to his major comments.

\section{Answers to the particular comments}

1. The reviewer mentions several particular solutions to the axisymmetric $N$-body problem. In 1996, I solved this problem in general formulation, and have obtained a complete solution to it. The solution included all particular solutions that by that time were known in the 
literature and, in addition, an infinite number of new, previously unknown solutions. Unfortunately, the reviewer failed to adequately comprehend this point. $\mathrm{He}$ cites the example of numerical solutions of the motion of several bodies in an orbit in form of figure of eight. This example is not relevant to the issue under consideration, as I have axisymmetric configurations of interaction of bodies, which are called central. Moreover, Carles Simo, the author of the paper, notes that the problem of finding the number of central configurations for a given $N$ and how it depends on the masses is still an open question.

2. The reviewer treats with distrust the statement, contained in my manuscript, that the Earth's axis makes oscillations of $\pm 7-8^{\circ}$ amplitude and that those oscillations define paleoclimate variations.

Also, the reviewer gives references to some other results reported in the literature. Based on those references, he arrives at quite paradoxical a conclusion that, first, my manuscript brings no new results and, second, that the results described in my manuscript are erroneous.

In my manuscript, I give proper reference to my previously published results and bring the reader's attention to publications where those results were substantiated. Had the reviewer taken an interest in adequately comprehending the matter, he would turn to reading my previous publications rather then casting doubts on my results and delivering conjectures. In my previous publications, I gave an exhaustive analysis to the problem as treated in other literature sources, including those mentioned by the reviewer, and had shown that the solutions proposed by the preceding authors were not fully adequate.

Unfortunately, the reviewer fails to comprehend the fact that, in science, no valid conclusions can be deduced from mere guesswork and assumptions.

3. Here, the reviewer cast doubt on my proof of the reason for additional rotation of Mercury's perihelion, which can be considered as one indicative of invalidity of the General relativity.

Here, the reviewer again arrives at quite contradictory a conclusion. On the one hand, he argues that this result is not a new one. On the other hand, he says that this result can be adequately understood within the Theory of general relativity, which has been known for one hundred years.

How cannot be new a result which rules out a Theory that has been known for one hundred years?

4. The reviewer was impressed by some points formulated in my manuscript in relation with globular star clusters and presently lacking an adequate explanation. He argues that such star clusters present a leading subject in contemporary astronomy to be tackled using advanced high-speed supercomputers.

But that is just the matter I have been engaged in. In my manuscript, I describe a new result that offers answers to the posed questions. On the contrary, the publications the reviewer mentions in his report give no such answers.
5. While trying to solve the problem regarding globular star clusters, I have constructed a spherically distributed $N$-bodies structure which for $N=100$ bodies proved to remain stable following 100 revolutions.

I was blamed by the reviewer for giving no proof to the result that the structure will remain stable over larger timescales $\left(10^{7}, 10^{8}\right.$ or $10^{9}$ revolutions). It should be noted that so far there is no evidence, apart from mine, that the Solar system, consisting of ten bodies (the Sun and nine planets), will remain stable over such timescales So the Reviewer's comment is ridiculous.

In this connection, consider another example. The first railway in Russia was constructed in 1837 to link together Petersburg and Tsarskoye Selo. How lucky the railway layers then were that, in those days, there were no critics to accuse them of not constructing an earth-round railway! Now, let us assume that such critics would have been in existence. Had they had a decisive influence on czar's opinion, there would have been, so far, no constructed railways in Russia at all.

Reverting to regular spherical structures, I would like to note that, in due course, all related points will be given proofs, and new Earth-like planets will certainly be created. That will be done despite the existence of critics like the reviewer, and despite the existence of people used to adhere to the opinion of such critics. Despite all this, there are other people like me, although few in number. Such people have always been bringing the truth and knowledge to the mankind and, later, their ideas were comprehended by many other rational thinkers.

6 . Here, the reviewer cast doubt on the possible ways toward solving the problem of building new Earth-like planets.

Apart from the problems mentioned by the reviewer, there exist many other problems, of which I make mention in my manuscript. In the manuscript, I propose a way toward solving this problem in succession, for instance, by making asteroids Earth's satellites.

To conclude, I would like to state that, unfortunately, the reviewer was definitely unable to comprehend the matter treated in my manuscript. The reviewer seems to be a kind of doctrinaire scientist, used to make science just by looking through literature. The article which I propose for publication in Springer Plus is intended for true, or pioneer, scientists, for people-creators. While reading (or reviewing) articles, such scientists never search for points to allow them blacken the author. In any article, a true scientist will find rational points that deserve attention to be put into practice.

\section{Answers to the major comments}

In his major comments, the reviewer tries to outline the general impression imposed on him by my manuscript "Advances in mechanics and outlook for further mankind progress". This is an impression made on a person familiar with ideal mathematical models who, however, considers such models in isolation from the surrounding world. The reviewer believes that the world functions by some physical laws unknown to him. 
On the contrary, in my article I show that recent achievements in mechanics provide a comprehensive explanation to the surrounding world and, the more so, they open up new opportunities both for its transformation and further mankind's progress. I have classified my article as a regular research paper because the article contains results obtained using acknowledged methods that were checked for adequacy in terms of all presently accepted criterions.

The reviewer has concluded the following:

1) my results and studies put in question the results of the "official" science;

2) among 25 cited publications, only three publications are not authored by me;

3 ) the manuscript therefore brings no new results.

These conclusions are contradictory. If some new results put in question previously reported results, they indeed present a novelty. Simultaneously, if new results have no analogues in literature, they need not be supplemented with any citations of previous publications.

In conclusion, I would like to state the following. The main argument produced by the reviewer and stating that my manuscript brings no new results is an erroneous one. There is no logic in the particular remarks delivered by the reviewer, or the reviewer shows up a bad command of the matter being discussed. Since the reviewer's report contains no evidence of errors in my manuscript, the report must be considered positive. If evaluated without bias, my article brings entirely new, fundamentally important results obviously deserving publication.

I, therefore, propose that my manuscript be published in Springer Plus together with the opinion expressed by the reviewer and with my reply to his comments.

The problems presently encountered by the mankind are due to the crisis of the establishment, or mainstream, science. There is no referee in the world which would be able to definitely tell us, in relation with the treated issues, whether we have arrived at valid or invalid conclusions. Invited to evaluate two opposite opinions produced, rationally thinking people will make a due choice for themselves. In this way, we shall cope with all problems the mankind presently faces.

\section{CONCLUSIONS}

Probably, it will take us more than several hundred years to create a new Earth. Today, we have enough space for all people on our Earth, and the situation will persist in the nearest future. The still unpopulated areas are the northern territories and the southern deserts. Sparsely populated rural areas are also to be populated. New thinking and new understanding of mankind's possibilities and tasks will make the meaninglessness of people's life in big cities quite obvious.

Today, we have enough space for all of us on our planet. Equally, we have much work to do and a plenty of tasks to accomplish for all of us. The purpose of science is to make each and everybody aware of this fact, and this will inevitably bring us nearer a real (not mythical!) paradise established on the Earth.

\section{ACKNOWLEDGMENT}

The numerical problems were solved on on the supercomputers of the Siberian Supercomputing Centre in the ICMMG SB RAS, Novosibirsk, Russia.

\section{REFERENCES}

[1] Nizovtsev, V.V. Principles of the Kinematic System of the World: Cartesian Alternative for the XXI Century Physics. Moscow, LIBROCOM Book House. 2013, 384 p. (In Russian).

[2] Smulsky, J.J. Main Path, Tyumen, 1993. (In Russian). http://samlib.ru/s/smulxskij_i_i/decdircdoc.shtml. ()

[3] Krendelev, F.P. Rapa-Nui (The Nub of the Universe). Novosibirsk: SB RAS Publishing House. 1996, 280 p. (In Russian).

[4] Smulsky, J.J. Non-relativistic picture of the world and brain ecology (relativity vs intelligence). in: Engineer's Brain in Action, edited by Acad. N.A. Malyushin, Tumen, GiproTumenNefteGaz, NefteGazProekt. 1997. Pp. 163175 (In Russian). http://samlib.ru/s/smulxskij_i_i/nerkar97.shtml.

[5] Smulsky, J.J. Impossible things can be made possible. Tumenskaya Pravda Segodnya. 1999. Iss. 38. (In Russian). http://samlib.ru/s/smulxskij_i_i/razgovor.shtml.

[6] Smulsky, J.J. Adequate understanding of the world as the basis for its optimal use. In: Collection of papers "Survival problems of post-socialistic Russia in the third millennium", Irkutsk, IrSTU, 2001, Iss. 2, Pt. 2, pp. 52 - 64 (In http://samlib.ru/s/smulxskij_i_i/pravpon1rtf.shtml. Russian).

[7] Smulsky, J.J. On the people's faith and sacred things. Novosibirsk. 2004. (In Russian). http://samlib.ru/s/smulxskij_i_i/versvnarhtm.shtml.

[8] Smulsky, J.J. The Theory of Interaction. Novosibirsk University, Scientific Publishing Center of UGG SB of RAS, Novosibirsk, 1999, 294 p. (In Russian). http://www.ikz.ru/ smulski/TVfulA5_2.pdf.

[9] Smulsky, J.J. The Theory of Interaction. PH Cultural Information Bank, Ekaterinburg, 2004, 302 p. http://www.ikz.ru/ smulski/TVEnA5_2.pdf.

[10] Smulsky, J.J. Axisymmetrical problem of gravitational interaction of N-bodies. Mathematical modeling, 2003. Vol. 15, No 5, pp. 27-36 (In Russian). http://www.ikz.ru/ smulski/smul1/Russian1/IntSunSyst/O svnb4.doc.

[11] Mel'nikov, V.P., Smulsky, J.J. and Smul'sky, Ya.I. Compound modeling of Earth rotation and possible implications for interaction of continents. Russian Geology and Geophysics, 2008, 49, pp. 851-858. http://www.ikz.ru/ smulski/Papers/RGG190.pdf.

[12] Smulsky, J.J. The Influence of the Planets, Sun and Moon on the Evolution of the Earth's Axis. International Journal of Astronomy and Astrophysics, 2011, 1, pp. 117-134. http://dx.doi.org/10.4236/ijaa.2011.13017.

[13] Smulsky, J.J. Analyzing the Lessons of the Development of the Orbital Theory of the Paleoclimate. Herald of the Russian Academy of Sciences. 2013, Vol. 83, No. 1, pp. 46-54 http://www.ikz.ru/ smulski/Papers/AnAstTP2E.pdf.

[14] Smulsky, J.J. Fundamental Principles and Results of a New Astronomic Theory of Climate Change. Advances in Astrophysics, 2016, Vol. 1, No. 1, 1-21 http://www.isaacpub.org/Journal/AdAp. 
[15] Smulsky J.J. New results on the Earth insolation and their correlation with the Late Pleistocene paleoclimate of West Siberia. Russian Geology and Geophysics, 2016, 57, 1099-1110. http://dx.doi.org/10.1016/j.rgg.2016.06.009.

[16] Smulsky J.J. Evolution of the Earth's axis and paleoclimate for 200 thousand years. Saarbrucken, Germany: "LAP Lambert Academic Publishing”. 2016, 228 p. ISBN 9783-659-95633-1. www.ikz.ru/ smulski/Papers/InfEvEAPC02MEn.pdf.

[17] Smulsky, J.J. New Components of the Mercury's Perihelion Precession. Natural Science, 2011, Vol. 3, No. 4, 268-274 http://dx.doi.org/10.4236/ns.2011.34034. http://www.scirp.org/journal/ns.

[18] Smulsky, J.J. Axisymmetric Coulomb Interaction and Research of its Stability by System Galactica. Open Access Library Journal, 2014, Vol. 1, e773, p. 1 - 23 http://dx.doi.org/10.4236/oalib.1100773.

[19] Smulsky, J.J. Multilayer Coulomb Structures: Mathematical Principia of Microcosm Mechanics. Open Access Library Journal, 2015, 2: e1661, 46 p. http://dx.doi.org/10.4236/oalib.1101661.

[20] Smulsky, J.J. Exact solution to the problem of $N$ bodies forming a multi-layer rotating structure. SpringerPlus, 2015, 4:361, pp. 1-16. DOI: 10.1186/s40064-015-1141-1. http://www.springerplus.com/content/4/1/361.

[21] Smulsky, J.J. The system of free access Galactica to compute interactions of $\mathrm{N}$ bodies. I. J. Modern Education and Computer Science, 2012, 11, pp. 1-20 DOI: 10.5815/ijmecs.2012.11.01.

[22] Smulsky J.J. Distributed structures on the sphere / Institute of the Earth Cryosphere SB RAS. - Tyumen. 43 p. Deposited in VINITI 22.08.2016, 2016, No. 112-V2016. http://www.ikz.ru/ smulski/Papers/SphDsSt2.pdf. (In Russian).

[23] Smulsky, J.J. and Smulsky, Ya.J. Dynamic Problems of the Planets and Asteroids, and Their Discussion. Intern.
Journal of Astronomy and Astrophysics, 2012, Vol. 2, No. 3, pp. 129-155. http://dx.doi.org/10.4236/ijaa.2012.23018.

[24] Smulsky J.J. and Smulsky Ya.J. Asteroids Apophis and 1950 DA: 1000 Years Orbit Evolution and Possible Use. In: Horizons in Earth Science Research, Vol. 6, Benjamin Veress and Jozsi Szigethy (editors), Nova Science Publishers, USA, 2012, pp. 63-97. https://www.novapublishers.com/catalog/index.php.

\section{Authors' Profiles}

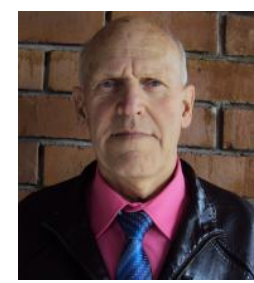

Joseph J. Smulsky was born in 1944. From 1967 to 1988 he worked in Research Institutes and Design bureaus of Novosibirsk. Since 1988 Prof. Joseph J. Smulsky has been working as chief scientist of the Institute of Earth Cryosphere. His research interests include a wide range of problems: foundations of mechanics and physics, vortex flow, air pollution, wind energy, Astronomical theory of climate change etc (http://wgalactica.ru/smul1/), some of which are presented in recent publications:

1. Smulsky, J.J. Fundamental Principles and Results of a New Astronomic Theory of Climate Change. Advances in Astrophysics, 2016, Vol. 1, No. 1, 1-21 http://www.isaacpub.org/Journal/AdAp.

2. Smulsky J.J. New results on the Earth insolation and their correlation with the Late Pleistocene paleoclimate of West Siberia. Russian Geology and Geophysics, 2016, 57, 1099-1110 http://dx.doi.org/10.1016/j.rgg.2016.06.009.

3. Smulsky J.J. Evolution of the Earth's axis and paleoclimate for 200 thousand years. Saarbrucken, Germany: "LAP Lambert Academic Publishing". 2016, 228 p. ISBN 978-3-659-956331. www.ikz.ru/ smulski/Papers/InfEvEAPC02MEn.pdf.

How to cite this paper: Joseph J. Smulsky,"Advances in Mechanics and Outlook for Future Mankind Progress", International Journal of Modern Education and Computer Science(IJMECS), Vol.9, No.1, pp.15-25, 2017.DOI: 10.5815/ijmecs.2017.01.02 